\author{
Nota científica \\ (Short Communication)
}

\title{
HERBIVORÍA EN CÍCADAS (CYCADOPHYTA) POR ADULTOS DE JANBECHYNEA ELONGATA JACOBY, 1888 (COLEOPTERA: ORSODACNIDAE) EN EL ANP SIERRA DE OTONTEPEC, VERACRUZ
}

Recibido: 27/10/2015; aceptado: 17/02/2016

Reyes-Ortiz, J. L., Luna-Ferrer, J. M., González-Gándara, C., Cruz-Morales G. E., Domínguez-Barradas, C. 2016. Feeding on cycads (Cycadophyta) by adults of Janbechynea elongata Jacoby, 1888 (Coleoptera: Orsodacnidae) in the ANP Sierra de Otontepec, Veracruz. Acta Zoológica Mexicana (n. s.), 31(2): 126-128.

\begin{abstract}
Little is known about the relationship between cycads and J. elongata. Since June 2009, adults J. elongata were observed feeding on Ceratozamia huastecorum in the Sierra de Otontepec; $C y$ cas revoluta and $C$. circinalis in public and private gardens in three locations in northern Veracruz. Its arrival is sudden and occurs at the end of spring. It presents gregarious or solitary behavior. It feeds on the leaflets with smooth texture and sometimes the rachis. Large populations of adult $J$. elongata occur in the morning and have been removed by the inhabitants to avoid damage to their cycads, this has disrupted the careful observation of their behavior and the possible meeting of larvae, pupae and eggs. Only $5 \%$ of the population $C$. huastecorum has been affected, while big Cycas revoluta and $C$. circinalis have been infested to $100 \%$.
\end{abstract}

Los coleópteros se alimentan y ovipositan sobre las hojas de una gran variedad de plantas (Ordóñez-Reséndiz et al., 2014). Son pocos los escarabajos especialistas que consumen raíces, tallos, frondas, estróbilos y semillas de las cícadas (Cycadophyta) (Castillo-Guevara \& Rico-Gray, 2003; Jolivet, 2005), entre estos, especies de Aulacoscelis y Janbechynea (Orsodacnidae) (Monrós, 1954; Suzuki \& Windsor, 1999; Windsor et al., 1999), conocidas por secuestrar las defensas químicas de las cícadas (Prado, 2011; Prado et al., 2011). El género Janbechynea se distribuye en Estados Unidos, México, Perú, Bolivia y Brasil (Windsor et al., 1999). Para México se reportan J. elongata, J. fulvipes J. georgepauljohnringo, J. julioi, J. maldonadoi, $J$. melyroides, J. snyderae, J. suzanita, J. virkkii y J. woodburyi (Santiago-Blay, 2004; Ordóñez-Reséndiz, 2014). Janbechynea elongata se ha registrado en Tamazunchale (S. L. P.), Playa Vicente (Ver.) (Cox y Windsor, 1999), y aquí se reporta para el Área Natural Protegida "Sierra de Otontepec" ubicada en el norte del estado de Veracruz. Monrós (1954) describió una fuerte infestación de J. elon- gata en cícadas importadas a EE. UU., sin especificar las especies (Windsor et al., 1999). Si bien no se ha establecido la relación con una planta hospedera en específico, su distribución coincide con la que presentan las familias Cycadaceae y Zamiaceae (Monrós, 1954).

Janbechynea elongata (Fig. 1a) fue observado por primera vez en Tancoco el 10 junio de 2009, después el 6 de julio de 2010 y el 16 de junio de 2011 entre las 13:00 y 16:00 horas. En total se encontraron cerca de 42 individuos adultos en grupos de 5 a 15, algunos se alimentaban de foliolos nuevos y el raquis de 12 individuos de $C$. huastecorum (Fig. 1b y c) (población de más de 100 individuos) otros solo descansaban. Por las condiciones del sitio, con pendientes de entre $70^{\circ}$ y $80^{\circ}$ se logró la captura de 16 insectos, estos se mantuvieron en laboratorio siguiendo la técnica de Windsor et al. (1999), se observó una copulación y, sobrevivieron cerca de tres semanas. En junio de 2011 y 2012 en Chontla, se reportaron enjambres de $J$. elongata, que llegaron a cinco ejemplares de $C$. revoluta y uno de $C$. circinalis. El 27 de junio de 2011, solo se observaron los restos de insectos muertos en $C$. revoluta (Fig. 1e) ya que fueron comidos por las hormigas. En uno de los jardines, las cícadas fueron retiradas, debido a que se consideraron marchitas. En junio de 2015, en Tantima y Chontla se reportaron enjambres de J. elongata que infestaron cerca de 20 individuos de C. revoluta y dos C. circinalis. De acuerdo con los residentes, cerca de ocho individuos de C. revoluta sufrieron un daño severo por este insecto (Fig. 1f), de no ser por la aplicación de insecticidas, el daño habría sido más fuerte. Janbechynea elongata llega volando, se esconde en el tronco y las frondas. Son frecuentes por la mañana siendo más activos al salir el sol. Prefieren las cícadas con diámetro de más de $1.50 \mathrm{~m}$. Siguen alimentándose de las frondas aun después de ser retiradas de la planta madre.

Este es el primer reporte sobre la alimentación de $J$. elongata, consumiendo frondas de Ceratozamia huastecorum (Zamiaceae), Cycas revoluta y C. circinalis 


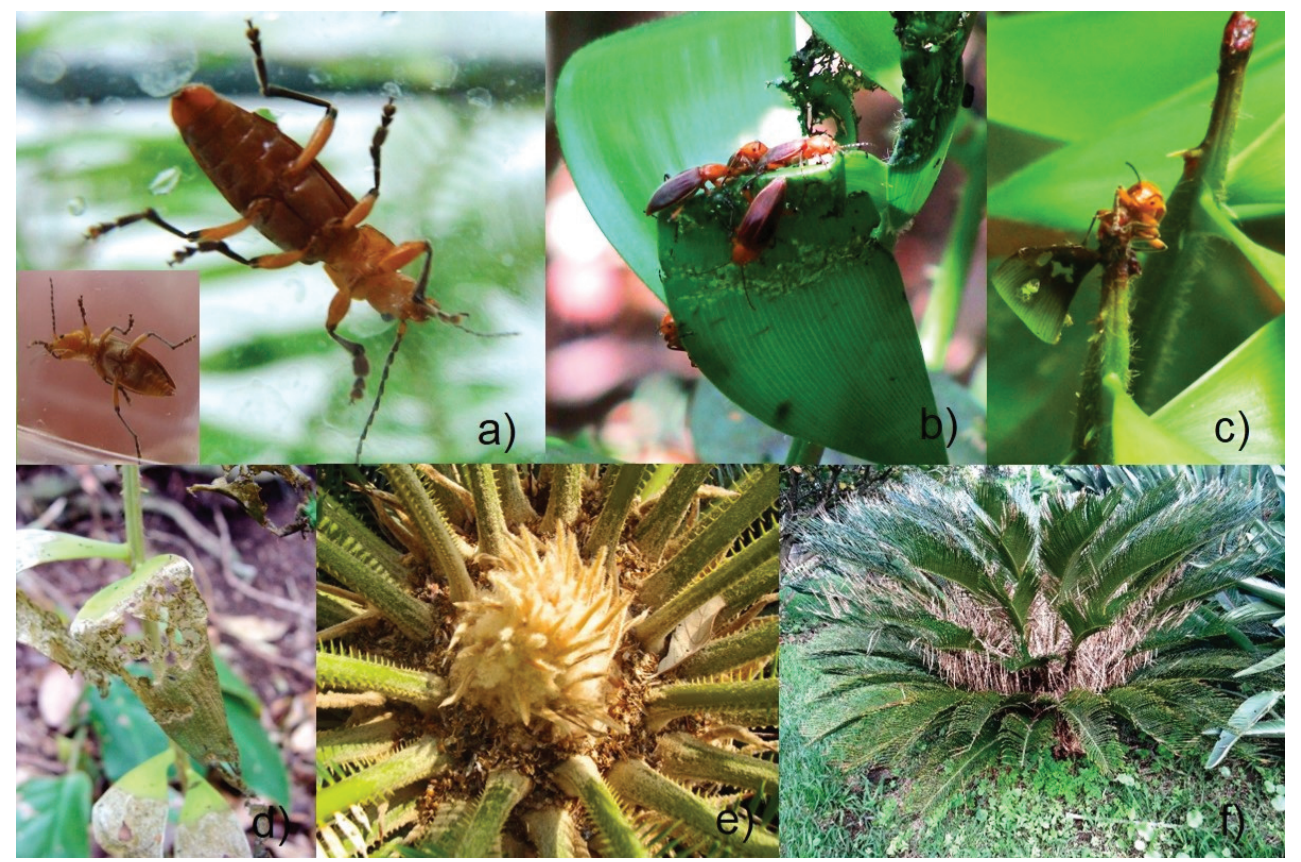

Figura 1. a) Adultos de Janbechynea elongata, nótese algunas diferencias en el abdomen. b) y c) Especímenes de $J$. elongata consumiendo foliolos nuevos y raquis de Ceratozamia huastecorum. d) Foliolos nuevos de C. huastecorum con un daño severo. e) Ejemplares de J. elongata muertos sobre un individuo de Cycas revoluta. f) C. revoluta de 1.70 de diámetro con un daño producido en menos de dos horas.

(Cycadaceae). La llegada del fitófago es a finales de la primavera y principios de verano durante la mañana, de acuerdo con Jolivet (2005) algunos Aulacoscelinae concurren en esta época del año. Presenta conducta gregaria o solitaria y aparecen de manera repentina. Estos hábitos son similares a los de Aulacoscelis sp. (Windsor et al., 1999). El daño que produce $J$. elongata a los foliolos nuevos es fuerte (Fig. 1d), las lesiones se asemejan a las producidas por Nomotus sp., que inducen a la marchitez y muerte de las frondas (Windsor et al., 1999). La distribución de $J$. elongata en la Sierra de Otontepec es entre los 200 y 700 msnm observándose en los municipios de Tancoco, Chontla y Tantima, en estos dos últimos, se reportaron enormes cantidades de este insecto. Las medidas para controlar a este herbívoro fueron sacrificarlos por medio de insecticidas y la poda completa de las cícadas. Windsor et al. (1999) señalan que es difícil observarlos sobre las cícadas pues su periodo de aparición es muy corto y sucede durante la noche o el atardecer, mientras que en el ANP sucede por la mañana y finaliza por la tarde.

Prado et al. $(2012,2014)$ sugieren que los coleópteros especialistas están sincronizados con el nuevo follaje de las cícadas por la textura suave de sus foliolos. Por otra parte, Windsor et al. (1999) mencionan que el estudio de los hábitos alimenticios de los Aulacoscelinae debe ser prioritario, por la amenaza que representan estos insectos para las cícadas. Además, Jolivet (2005) plantea que la ovoposición, las larvas y pupas se llevan a cabo dentro de los estróbilos y las semillas, pero esto no ha sido demostrado. En este sentido, puede convertirse en un problema para la preservación de las cícadas, por los enjambres de $J$. elongata que llegan de manera repentina y en pocas horas causan un daño muy fuerte. La presencia de pocos individuos de J. elongata en C. huastecorum está influenciada por su ubicación en la sierra (a $900 \mathrm{msnm}$ ) y que no está expuesta a campo abierto como $C$. revoluta y $C$. circinalis (a $200 \mathrm{msnm}$ ).

AGRADECIMIENTOS. Al editor y revisores, a la Dra. Ordóñez-Reséndiz (UNAM), al Dr. Windsor y al Dr. Santiago-Blay (Smithsonian Tropical Research Institute) por sus valiosos comentarios. A los habitantes de Tantima y Chontla por su apoyo en campo.

\section{LITERATURA CITADA}

Castillo-Guevara, C. \& Rico-Gray, V. 2003. The role of macrozamine and cycasin in cycads (Cycadales) as antiherbivore defenses. Journal of the Torrey Botanical Society, 130: 206-217. 
Reyes-Ortiz et al.: Herbivoría en cícadas por Janbechynea elongata

Cox, M. L. \& Windsor, D. M. 1999. The first instar larva of $A u$ lacoscelis appendiculata n. sp. (Coleoptera: Chrysomelidae: Aulacoscelinae) and its value in the placement of the Aulacoscelinae. Journal of Natural History, 33: 1049-1087.

Jolivet, P. 2005. Cycads and beetles: recent views on pollination. The Cycad Newsletter, 28(3-4): 3-7.

Monrós, F. 1954. Revision of the crysomelid subfamily Aulacoscelinae. Bulletin of the Museum of Comparative Zoology, 112: 321360.

Ordóñez-Reséndiz, M. M. 2014. Catálogo de Autoridades Taxonómicas y base de datos curatorial de la familia Chrysomelidae en México. Universidad Nacional Autónoma de México. Facultad de Estudios Superiores Zaragoza. Informe final, SNIB-CONABIO. Proyecto No. HS003. México, D. F.

Ordóñez-Reséndiz, M. M., López-Pérez, S. \& Rodríguez-Mirón G. 2014. Biodiversidad de Chrysomelidae (Coleoptera) en México. Revista Mexicana de Biodiversidad, 85: 271-278.

Prado, A. 2011. The cycad herbivores. Bulletin de la Société d'entomologie du Quebec, 18: 3-6.

Prado, A., Ledezma, J., Cubilla-Rios, L., Bede, J. C. \& Windsor, D. M. 2011. Two genera of Aulacoscelinae beetles reflexively bleed azoxyglycosides found in their host cycads. Journal of Chemical Ecological, 37: 736-740.

Prado, A., Mckenna D. D. \& Windsor, D. 2012. Molecular evidence of cycad seed predation by inmature Aulacoscelidinae (Coleoptera: Orsodacnidae). Systematic Entomology, 37: 747-757.

Prado, A., Sierra, A., Windsor, D. \& Bede, J. C. 2014. Leaf traits and herbivory level in a tropical gimnosperma, Zamia stevensonii (Zamiaceae). American Journal Botany, 101: 1-11.

Santiago-Blay, J. A. 2004. Description of seven new species of Janbechynea (Orsodacnidae or Chrysomelidae, Sensu lato) from Mexico: Availability of new names and corrections. Entomological News, 115: 108-112.

Suzuki, K. \& Windsor, D. M. 1999. The internal reproductive system of Panamanian Aulacoscelis sp. (Coleoptera: Chrysomelidae, Aulacoscelinae) and comments on the systematic position of the subfamily. Entomological Science, 2: 391-398.

Windsor, D., Ness J., Gomez, L. D. \& Jolivet, P. 1999. Species of Aulacoscelis Duponchal and Chevrolat (Chrysomelidae) and Nomotus Gorham (Languriidae) feed on fronds of Central American cycads. Coleopterists Bulletin, 53: 217-231.

\section{José LuIS REYES-ORTIZ*, José MANuel LUNA- FERRER, CARLOS GONZÁLEZ-GÁNDARA, GERARDo Eliseo CRUZ-MORALES \& CONSUELO DOMÍNGUEZ-BARRADAS}

Facultad de Ciencias Biológicas y Agropecuarias, Campus Tuxpan. Universidad Veracruzana. Carretera Tuxpan-Tampico Km 7.5, Col. Universitaria, C. P. 92895, Tuxpan, Veracruz.

*jlrouv@hotmail.com; bien_sobres@hotmail.com; cggandara@hotmail.com; jerry_cm00@hotmail.com; consuelodb66@hotmail.com. 